\title{
Noncommutativity of Lepton Mass Matrices: Flavor Mixing and CP Violation
}

\author{
Zhi-zhong Xing \\ Institute of High Energy Physics, P.O. Box 918 (4), Beijing 100039, China \\ E-mail: xingzz@mail.ihep.ac.cn
}

\begin{abstract}
I follow a lesson learnt from Heisenberg's matrix Quantum Mechanics to study the property of lepton flavor mixing and $\mathrm{CP}$ violation. I show that the commutator of lepton mass matrices defined in vacuum keeps invariant under terrestrial matter effects within the four-neutrino mixing scheme. A set of model-independent sum rules for neutrino masses are obtained, and they can be generalized to hold for an arbitrary number of neutrino families. Useful sum rules for the rephasing-invariant measures of leptonic $\mathrm{CP}$ violation have also been found. Finally I present a generic formula of T-violating asymmetries applicable to the future long-baseline neutrino oscillation experiments.
\end{abstract}

A lesson learnt from Heisenberg's matrix Quantum Mechanics is that the observables are represented by Hermitian operators and their commutator yields a description of their compatibility, i.e., whether the observables can be measured simultaneously or not. As already noticed by some authors [i], the commutator of up- and down-quark mass matrices describes whether they can be diagonalized simultaneously or not. The noncommutativity of fermion mass matrices in mathematics is indeed a consequence of flavor mixing and $\mathrm{CP}$ violation in physics. In this talk I am going to demonstrate that the commutator of lepton mass matrices is particularly useful to establish the realtions between the observables of neutrino mixing in vacuum and those in matter.

The motivation to study lepton flavor mixing comes, first of all, from the robust SuperKamiokande evidence for atmospheric and solar neutrino oscillations [2]. In addition, the $\nu_{\mu} \rightarrow \nu_{e}$ oscillation has been observed by the LSND Collaboration [3]. A simultaneous interpretation of solar, atmospheric and LSND neutrino oscillation data has to invoke the existence of a light sterile neutrino [i] , because they involve three distinct masssquared differences $\left(\Delta m_{\mathrm{sun}}^{2} \ll \Delta m_{\mathrm{atm}}^{2} \ll \Delta m_{\mathrm{LSND}}^{2}\right)$. In the four-neutrino mixing scheme, $\mathrm{CP}$ violation is generally expected to manifest itself. To measure leptonic CP- and Tviolating effects needs a new generation of accelerator neutrino experiments with very long baselines [5]. In such long-baseline experiments the terrestrial matter effects, which are likely to deform the neutrino oscillation patterns in vacuum and to fake the genuine $\mathrm{CP}$ - and T-violating signals, must be taken into account. To pin down the underlying dynamics of lepton mass generation and $\mathrm{CP}$ violation relies crucially upon how accurately the fundamental parameters of lepton flavor mixing can be measured and disentangled from matter effects [6]. It is therefore desirable to explore possible model-independent relations between the effective neutrino masses in matter and the genuine neutrino masses 
in vacuum. It is also useful to establish some model-independent relations between the rephasing-invariant measures of $\mathrm{CP}$ violation in matter and those in vacuum [7].

This talk aims to show that the commutator of lepton mass matrices is invariant under terrestrial matter effects. As a consequence, some concise sum rules of neutrino masses can be obtained model-independently. Such sum rules can even be generalized to hold for an arbitrary number of neutrino families. Another set of sum rules are derived as well for the rephasing-invariant measures of leptonic $\mathrm{CP}$ violation in matter. Finally I present a generic formula of T-violating asymmetries, which is applicable in particular to the future long-baseline neutrino oscillation experiments.

The phenomenon of lepton flavor mixing arises from the mismatch between the diagonalization of the charged lepton mass matrix $M_{l}$ and that of the neutrino mass matrix $M_{\nu}$ in an arbitrary flavor basis. Without loss of generality, one may choose to identify the flavor eigenstates of charged leptons with their mass eigenstates. In this specific basis, where $M_{l}$ is diagonal, the lepton flavor mixing matrix $V$ links the neutrino flavor eigenstates directly to the neutrino mass eigenstates. For the admixture of one sterile $\left(\nu_{s}\right)$ and three active $\left(\nu_{e}, \nu_{\mu}, \nu_{\tau}\right)$ neutrinos, the explicit form of $V$ reads

$$
\left(\begin{array}{c}
\nu_{s} \\
\nu_{e} \\
\nu_{\mu} \\
\nu_{\tau}
\end{array}\right)=\left(\begin{array}{cccc}
V_{s 0} & V_{s 1} & V_{s 2} & V_{s 3} \\
V_{e 0} & V_{e 1} & V_{e 2} & V_{e 3} \\
V_{\mu 0} & V_{\mu 1} & V_{\mu 2} & V_{\mu 3} \\
V_{\tau 0} & V_{\tau 1} & V_{\tau 2} & V_{\tau 3}
\end{array}\right)\left(\begin{array}{c}
\nu_{0} \\
\nu_{1} \\
\nu_{2} \\
\nu_{3}
\end{array}\right),
$$

where $\nu_{i}$ (for $i=0,1,2,3$ ) denote the mass eigenstates of four neutrinos. The effective Hamiltonian responsible for the propagation of neutrinos in vacuum can be written as

$$
\mathcal{H}_{\mathrm{eff}}=\frac{1}{2 E}\left(M_{\nu} M_{\nu}^{\dagger}\right)=\frac{1}{2 E}\left(V D_{\nu}^{2} V^{\dagger}\right)
$$

where $D_{\nu} \equiv \operatorname{Diag}\left\{m_{0}, m_{1}, m_{2}, m_{3}\right\}, m_{i}$ are the neutrino mass eigenvalues, and $E \gg$ $m_{i}$ denotes the neutrino beam energy. When active neutrinos travel through a normal material medium (e.g., the earth), which consists of electrons but of no muons or taus, they encounter both charged- and neutral-current interactions with electrons. The neutralcurrent interaction is universal for $\nu_{e}, \nu_{\mu}$ and $\nu_{\tau}$, while the charged-current interaction is associated only with $\nu_{e}$. Their effects on the mixing and propagating features of neutrinos have to be taken into account in all long-baseline neutrino oscillation experiments. Let us use $\tilde{M}_{\nu}$ and $\tilde{V}$ to denote the effective neutrino mass matrix and the effective flavor mixing matrix in matter, respectively. Then the effective Hamiltonian responsible for the propagation of neutrinos in matter can be written as

$$
\tilde{\mathcal{H}}_{\mathrm{eff}}=\frac{1}{2 E}\left(\tilde{M}_{\nu} \tilde{M}_{\nu}^{\dagger}\right)=\frac{1}{2 E}\left(\tilde{V} \tilde{D}_{\nu}^{2} \tilde{V}^{\dagger}\right)
$$

where $\tilde{D}_{\nu} \equiv \operatorname{Diag}\left\{\tilde{m}_{0}, \tilde{m}_{1}, \tilde{m}_{2}, \tilde{m}_{3}\right\}$, and $\tilde{m}_{i}$ are the effective neutrino mass eigenvalues in matter. The deviation of $\tilde{\mathcal{H}}_{\text {eff }}$ from $\mathcal{H}_{\text {eff }}$ is given by

$$
\Delta \mathcal{H}_{\text {eff }} \equiv \tilde{\mathcal{H}}_{\text {eff }}-\mathcal{H}_{\text {eff }}=\left(\begin{array}{cccc}
a^{\prime} & 0 & 0 & 0 \\
0 & a & 0 & 0 \\
0 & 0 & 0 & 0 \\
0 & 0 & 0 & 0
\end{array}\right)
$$

where $a=\sqrt{2} G_{\mathrm{F}} N_{e}$ and $a^{\prime}=\sqrt{2} G_{\mathrm{F}} N_{n} / 2$ with $N_{e}$ and $N_{n}$ being the background densities of electrons and neutrons [8], respectively. 
Now let me introduce the commutators of $4 \times 4$ lepton mass matrices to describe the flavor mixing of one sterile and three active neutrinos. Without loss of any generality, I continue to work in the afore-chosen flavor basis, where $M_{l}$ takes the diagonal form $D_{l}=\operatorname{Diag}\left\{m_{s}, m_{e}, m_{\mu}, m_{\tau}\right\}$ with $m_{s}=0$. Note that I have assumed the $(1,1)$ element of $D_{l}$ to be zero, because there is no counterpart of the sterile neutrino $\nu_{s}$ in the charged lepton sector. We shall see later on that our physical results are completely independent of $m_{s}$, no matter what value it may take. The commutator of lepton mass matrices in vacuum and that in matter can then be defined as

$$
\begin{aligned}
& C \equiv i\left[M_{\nu} M_{\nu}^{\dagger}, M_{l} M_{l}^{\dagger}\right]=i\left[V D_{\nu}^{2} V^{\dagger}, D_{l}^{2}\right], \\
& \tilde{C} \equiv i\left[\tilde{M}_{\nu} \tilde{M}_{\nu}^{\dagger}, M_{l} M_{l}^{\dagger}\right]=i\left[\tilde{V} \tilde{D}_{\nu}^{2} \tilde{V}^{\dagger}, D_{l}^{2}\right] .
\end{aligned}
$$

Obviously $C$ and $\tilde{C}$ are traceless Hermitian matrices. In terms of neutrino masses and flavor mixing matrix elements, I obtain the explicit expressions of $C$ and $\tilde{C}$ as follows:

$$
\begin{aligned}
C & =i\left(\begin{array}{cccc}
0 & \Delta_{e s} Z_{s e} & \Delta_{\mu s} Z_{s \mu} & \Delta_{\tau s} Z_{s \tau} \\
\Delta_{s e} Z_{e s} & 0 & \Delta_{\mu e} Z_{e \mu} & \Delta_{\tau e} Z_{e \tau} \\
\Delta_{s \mu} Z_{\mu s} & \Delta_{e \mu} Z_{\mu e} & 0 & \Delta_{\tau \mu} Z_{\mu \tau} \\
\Delta_{s \tau} Z_{\tau s} & \Delta_{e \tau} Z_{\tau e} & \Delta_{\mu \tau} Z_{\tau \mu} & 0
\end{array}\right), \\
\tilde{C} & =i\left(\begin{array}{cccc}
0 & \Delta_{e s} \tilde{Z}_{s e} & \Delta_{\mu s} \tilde{Z}_{s \mu} & \Delta_{\tau s} \tilde{Z}_{s \tau} \\
\Delta_{s e} \tilde{Z}_{e s} & 0 & \Delta_{\mu e} \tilde{Z}_{e \mu} & \Delta_{\tau e} \tilde{Z}_{e \tau} \\
\Delta_{s \mu} \tilde{Z}_{\mu s} & \Delta_{e \mu} \tilde{Z}_{\mu e} & 0 & \Delta_{\tau \mu} \tilde{Z}_{\mu \tau} \\
\Delta_{s \tau} \tilde{Z}_{\tau s} & \Delta_{e \tau} \tilde{Z}_{\tau e} & \Delta_{\mu \tau} \tilde{Z}_{\tau \mu} & 0
\end{array}\right),
\end{aligned}
$$

where $\Delta_{\alpha \beta} \equiv m_{\alpha}^{2}-m_{\beta}^{2}$ for $\alpha \neq \beta$ running over $(s, e, \mu, \tau)$, and

$$
Z_{\alpha \beta} \equiv \sum_{i=0}^{3}\left(m_{i}^{2} V_{\alpha i} V_{\beta i}^{*}\right), \quad \tilde{Z}_{\alpha \beta} \equiv \sum_{i=0}^{3}\left(\tilde{m}_{i}^{2} \tilde{V}_{\alpha i} \tilde{V}_{\beta i}^{*}\right)
$$

One can see that $\Delta_{\beta \alpha}=-\Delta_{\alpha \beta}, Z_{\beta \alpha}=Z_{\alpha \beta}^{*}$ and $\tilde{Z}_{\beta \alpha}=\tilde{Z}_{\alpha \beta}^{*}$ hold.

To find out how $\tilde{Z}_{\alpha \beta}$ is connected with $Z_{\alpha \beta}$, I need to establish the relation between $\tilde{C}$ and $C$. Taking Eqs. (2), (3) and (4) into account, I immediately obtain

$$
\tilde{C}=2 i E\left[\tilde{\mathcal{H}}_{\mathrm{eff}}, D_{l}^{2}\right]=C+2 i E\left[\Delta \mathcal{H}_{\mathrm{eff}}, D_{l}^{2}\right]=C
$$

This interesting result indicates that the commutator of lepton mass matrices in vacuum is invariant under terrestrial matter effects. As a straightforward consequence of $\tilde{C}=C$, I arrive at $\tilde{Z}_{\alpha \beta}=Z_{\alpha \beta}$ from Eq. (6). Then a set of concise sum rules of neutrino masses emerge [7]:

$$
\sum_{i=0}^{3}\left(\tilde{m}_{i}^{2} \tilde{V}_{\alpha i} \tilde{V}_{\beta i}^{*}\right)=\sum_{i=0}^{3}\left(m_{i}^{2} V_{\alpha i} V_{\beta i}^{*}\right)
$$

or equivalently

$$
\sum_{i=1}^{3}\left(\tilde{\Delta}_{i 0} \tilde{V}_{\alpha i} \tilde{V}_{\beta i}^{*}\right)=\sum_{i=1}^{3}\left(\Delta_{i 0} V_{\alpha i} V_{\beta i}^{*}\right)
$$

where $\Delta_{i 0} \equiv m_{i}^{2}-m_{0}^{2}$ and $\tilde{\Delta}_{i 0} \equiv \tilde{m}_{i}^{2}-\tilde{m}_{0}^{2}$ for $i=1,2,3$. It becomes obvious that the validity of Eq. (9) or Eq. (10) has nothing to do with the assumption of $m_{s}=0$ in 
the charged lepton sector. Although I have derived these sum rules in the four-neutrino mixing scheme, they may simply be generalized to hold for an arbitrary number of neutrino families.

It should be noted that $Z_{\alpha \beta}$ and $\tilde{Z}_{\alpha \beta}$ are sensitive to a redefinition of the phases of charged lepton fields. The simplest rephasing-invariant equality is of course $\left|\tilde{Z}_{\alpha \beta}\right|=\left|Z_{\alpha \beta}\right|$. For the description of $\mathrm{CP}$ or $\mathrm{T}$ violation in neutrino oscillations, we are more interested in the following rephasing-invariant relationship:

$$
\tilde{Z}_{\alpha \beta} \tilde{Z}_{\beta \gamma} \tilde{Z}_{\gamma \alpha}=Z_{\alpha \beta} Z_{\beta \gamma} Z_{\gamma \alpha}
$$

for $\alpha \neq \beta \neq \gamma$ running over $(s, e, \mu, \tau)$. As one can see later on, the imaginary parts of $Z_{\alpha \beta} Z_{\beta \gamma} Z_{\gamma \alpha}$ and $\tilde{Z}_{\alpha \beta} \tilde{Z}_{\beta \gamma} \tilde{Z}_{\gamma \alpha}$ are related respectively to leptonic CP violation in vacuum and that in matter.

It should also be noted that the results obtained above are only valid for neutrinos propagating in vacuum and in matter. As for antineutrinos, the corresponding formulas can straightforwardly be written out from Eqs. (3) - (11) through the replacements $V \Longrightarrow V^{*}, a \Longrightarrow-a$ and $a^{\prime} \Longrightarrow-a^{\prime}$.

In terms of the matrix elements of $V$ or $\tilde{V}$, one may define the rephasing-invariant measures of $\mathrm{CP}$ violation as follows:

$$
J_{\alpha \beta}^{i j} \equiv \operatorname{Im}\left(V_{\alpha i} V_{\beta j} V_{\alpha j}^{*} V_{\beta i}^{*}\right), \quad \tilde{J}_{\alpha \beta}^{i j} \equiv \operatorname{Im}\left(\tilde{V}_{\alpha i} \tilde{V}_{\beta j} \tilde{V}_{\alpha j}^{*} \tilde{V}_{\beta i}^{*}\right),
$$

where the Greek subscripts $(\alpha \neq \beta)$ run over $(s, e, \mu, \tau)$, and the Latin superscripts $(i \neq j)$ run over $(0,1,2,3)$. Of course, $J_{\alpha \beta}^{i i}=J_{\alpha \alpha}^{i j}=0$ and $\tilde{J}_{\alpha \beta}^{i i}=\tilde{J}_{\alpha \alpha}^{i j}=0$ hold by definition. With the help of the unitarity of $V$ or $\tilde{V}$, one may straightforwardly obtain some correlation equations of $J_{\alpha \beta}^{i j}$ and $\tilde{J}_{\alpha \beta}^{i j}$ [9]. Therefore there are only nine independent $J_{\alpha \beta}^{i j}$ (or $\tilde{J}_{\alpha \beta}^{i j}$ ) in the four-neutrino mixing scheme under discussion. If only the flavor mixing of three active neutrinos is taken into account, there will be a single independent $J_{\alpha \beta}^{i j}$ (or $\tilde{J}_{\alpha \beta}^{i j}$ ), redefined as $J$ (or $\tilde{J}$ ). It is a unique feature of the three-family flavor mixing scenario, for either leptons or quarks [1], that there exists a universal CP-violating parameter.

To establish the relation between $\tilde{J}_{\alpha \beta}^{i j}$ and $J_{\alpha \beta}^{i j}$, I make use of th equality in Eq. (11). The key point is that the imaginary parts of the rephasing-invariant quantities $Z_{\alpha \beta} Z_{\beta \gamma} Z_{\gamma \alpha}$ and $\tilde{Z}_{\alpha \beta} \tilde{Z}_{\beta \gamma} \tilde{Z}_{\gamma \alpha}$,

$$
\begin{aligned}
& \operatorname{Im}\left(Z_{\alpha \beta} Z_{\beta \gamma} Z_{\gamma \alpha}\right)=\sum_{i=1}^{3} \sum_{j=1}^{3} \sum_{k=1}^{3}\left[\Delta_{i 0} \Delta_{j 0} \Delta_{k 0} \operatorname{Im}\left(V_{\alpha i} V_{\beta j} V_{\gamma k} V_{\alpha k}^{*} V_{\beta i}^{*} V_{\gamma j}^{*}\right)\right] \\
& \operatorname{Im}\left(\tilde{Z}_{\alpha \beta} \tilde{Z}_{\beta \gamma} \tilde{Z}_{\gamma \alpha}\right)=\sum_{i=1}^{3} \sum_{j=1}^{3} \sum_{k=1}^{3}\left[\tilde{\Delta}_{i 0} \tilde{\Delta}_{j 0} \tilde{\Delta}_{k 0} \operatorname{Im}\left(\tilde{V}_{\alpha i} \tilde{V}_{\beta j} \tilde{V}_{\gamma k} \tilde{V}_{\alpha k}^{*} \tilde{V}_{\beta i}^{*} \tilde{V}_{\gamma j}^{*}\right)\right]
\end{aligned}
$$

which do not vanish unless leptonic $\mathrm{CP}$ and $\mathrm{T}$ are good symmetries, amount to each other. The right-hand side of Eq. (13) can be expanded in terms of $J_{\alpha \beta}^{i j}$ and $\tilde{J}_{\alpha \beta}^{i j}$. In doing so, one needs to use Eq. (12) as well as the unitarity conditions of $V$ and $\tilde{V}$ frequently. After some lengthy but straightforward algebraic calculations, I arrive at the following sum rules of $\mathrm{CP}$ - or T-violating parameters:

$$
\begin{aligned}
& \tilde{\Delta}_{10} \tilde{\Delta}_{20} \tilde{\Delta}_{30} \sum_{i=1}^{3}\left(\tilde{J}_{\alpha \beta}^{0 i}\left|\tilde{V}_{\gamma i}\right|^{2}+\tilde{J}_{\beta \gamma}^{0 i}\left|\tilde{V}_{\alpha i}\right|^{2}+\tilde{J}_{\gamma \alpha}^{0 i}\left|\tilde{V}_{\beta i}\right|^{2}\right) \\
& +\sum_{i=1}^{3} \sum_{j=1}^{3}\left[\tilde{\Delta}_{i 0} \tilde{\Delta}_{j 0}^{2}\left(\tilde{J}_{\alpha \beta}^{i j}\left|\tilde{V}_{\gamma j}\right|^{2}+\tilde{J}_{\beta \gamma}^{i j}\left|\tilde{V}_{\alpha j}\right|^{2}+\tilde{J}_{\gamma \alpha}^{i j}\left|\tilde{V}_{\beta j}\right|^{2}\right)\right]
\end{aligned}
$$




$$
\begin{aligned}
= & \Delta_{10} \Delta_{20} \Delta_{30} \sum_{i=1}^{3}\left(J_{\alpha \beta}^{0 i}\left|V_{\gamma i}\right|^{2}+J_{\beta \gamma}^{0 i}\left|V_{\alpha i}\right|^{2}+J_{\gamma \alpha}^{0 i}\left|V_{\beta i}\right|^{2}\right) \\
& +\sum_{i=1}^{3} \sum_{j=1}^{3}\left[\Delta_{i 0} \Delta_{j 0}^{2}\left(J_{\alpha \beta}^{i j}\left|V_{\gamma j}\right|^{2}+J_{\beta \gamma}^{i j}\left|V_{\alpha j}\right|^{2}+J_{\gamma \alpha}^{i j}\left|V_{\beta j}\right|^{2}\right)\right] .
\end{aligned}
$$

I would like to remark that this result is model-independent and rephasing-invariant. It may be considerably simplified, once the hierarchy of neutrino masses and that of flavor mixing angles are theoretically assumed or experimentally measured. If one "switches off" the mass of the sterile neutrino and its mixing with active neutrinos (i.e., $a^{\prime}=0$, $\Delta_{i 0}=m_{i}^{2}, \tilde{\Delta}_{i 0}=\tilde{m}_{i}^{2}, J_{\alpha \beta}^{0 i}=0$, and $\tilde{J}_{\alpha \beta}^{0 i}=0$ ), then Eq. (14) turns out to take the form $\tilde{J} \tilde{\Delta}_{21} \tilde{\Delta}_{31} \tilde{\Delta}_{32}=J \Delta_{21} \Delta_{31} \Delta_{32}$. This elegant relationship has been derived in Refs. [7, [10] with the help of the equality $\operatorname{Det}(\tilde{C})=\operatorname{Det}(C)$, instead of Eq. (11), in the three-neutrino mixing scheme.

The matter-corrected CP-violating parameters $\tilde{J}_{\alpha \beta}^{i j}$ can, at least in principle, be determined from the measurement of CP- and T-violating effects in a variety of long-baseline neutrino oscillation experiments. The conversion probability of a neutrino $\nu_{\alpha}$ to another neutrino $\nu_{\beta}$ is given in matter as

$$
\tilde{P}\left(\nu_{\alpha} \rightarrow \nu_{\beta}\right)=\delta_{\alpha \beta}-4 \sum_{i<j}\left[\operatorname{Re}\left(\tilde{V}_{\alpha i} \tilde{V}_{\beta j} \tilde{V}_{\alpha j}^{*} \tilde{V}_{\beta i}^{*}\right) \sin ^{2} \tilde{F}_{j i}\right]-2 \sum_{i<j}\left[\tilde{J}_{\alpha \beta}^{i j} \sin \left(2 \tilde{F}_{j i}\right)\right],
$$

where $\tilde{F}_{j i} \equiv 1.27 \tilde{\Delta}_{j i} L / E$ with $\tilde{\Delta}_{j i} \equiv \tilde{m}_{j}^{2}-\tilde{m}_{i}^{2}, L$ stands for the baseline length (in unit of $\mathrm{km}$ ), and $E$ is the neutrino beam energy (in unit of $\mathrm{GeV}$ ). The transition probability $\tilde{P}\left(\nu_{\beta} \rightarrow \nu_{\alpha}\right)$ can directly be read off from Eq. (15), if the replacements $\tilde{J}_{\alpha \beta}^{i j} \Longrightarrow-\tilde{J}_{\alpha \beta}^{i j}$ are made. To obtain the probability $\tilde{P}\left(\bar{\nu}_{\alpha} \rightarrow \bar{\nu}_{\beta}\right)$, however, both the replacements $J_{\alpha \beta}^{i j} \Longrightarrow$ $-J_{\alpha \beta}^{i j}$ and $\left(a, a^{\prime}\right) \Longrightarrow\left(-a,-a^{\prime}\right)$ need be made for Eq. (15). In this case, $\tilde{P}\left(\bar{\nu}_{\alpha} \rightarrow \bar{\nu}_{\beta}\right)$ is not equal to $\tilde{P}\left(\nu_{\beta} \rightarrow \nu_{\alpha}\right)$. The difference between $\tilde{P}\left(\bar{\nu}_{\alpha} \rightarrow \bar{\nu}_{\beta}\right)$ and $\tilde{P}\left(\nu_{\beta} \rightarrow \nu_{\alpha}\right)$ is a false signal of CPT violation, induced actually by the matter effect [6]. Thus the CP-violating asymmetries between $\tilde{P}\left(\nu_{\alpha} \rightarrow \nu_{\beta}\right)$ and $\tilde{P}\left(\bar{\nu}_{\alpha} \rightarrow \bar{\nu}_{\beta}\right)$ are in general different from the Tviolating asymmetries between $\tilde{P}\left(\nu_{\alpha} \rightarrow \nu_{\beta}\right)$ and $\tilde{P}\left(\nu_{\beta} \rightarrow \nu_{\alpha}\right)$. The latter can be explicitly expressed as follows:

$$
\begin{aligned}
\Delta \tilde{P}_{\alpha \beta} \equiv & \tilde{P}\left(\nu_{\beta} \rightarrow \nu_{\alpha}\right)-\tilde{P}\left(\nu_{\alpha} \rightarrow \nu_{\beta}\right) \\
= & 4\left[\tilde{J}_{\alpha \beta}^{01} \sin \left(2 \tilde{F}_{10}\right)+\tilde{J}_{\alpha \beta}^{02} \sin \left(2 \tilde{F}_{20}\right)+\tilde{J}_{\alpha \beta}^{03} \sin \left(2 \tilde{F}_{30}\right)\right. \\
& \left.+\tilde{J}_{\alpha \beta}^{12} \sin \left(2 \tilde{F}_{21}\right)+\tilde{J}_{\alpha \beta}^{13} \sin \left(2 \tilde{F}_{31}\right)+\tilde{J}_{\alpha \beta}^{23} \sin \left(2 \tilde{F}_{32}\right)\right] .
\end{aligned}
$$

If the hierarchical patterns of neutrino masses and flavor mixing angles are assumed, the expression of $\Delta \tilde{P}_{\alpha \beta}$ may somehow be simplified [11]. Note that only three of the twelve nonvanishing asymmetries $\Delta \tilde{P}_{\alpha \beta}$ are independent, as a consequence of the unitarity of $\tilde{V}$ or the correlation of $\tilde{J}_{\alpha \beta}^{i j}$. Since only the transition probabilities of active neutrinos can be realistically measured, we are more interested in the T-violating asymmetries $\Delta \tilde{P}_{e \mu}$, $\Delta \tilde{P}_{\mu \tau}$ and $\Delta \tilde{P}_{\tau e}$. These three measurables, which are independent of one another in the four-neutrino mixing scheme under discussion, must be identical in the conventional three-neutrino mixing scheme. In the latter case, where $a^{\prime}=0, \tilde{J}_{\alpha \beta}^{01}=\tilde{J}_{\alpha \beta}^{02}=\tilde{J}_{\alpha \beta}^{03}=0$, and $\tilde{J}_{\alpha \beta}^{12}=-\tilde{J}_{\alpha \beta}^{13}=\tilde{J}_{\alpha \beta}^{23}=\tilde{J}$ for $(\alpha, \beta)$ running over $(e, \mu),(\mu, \tau)$ and $(\tau, e)$, Eq. (16) can be simplified to $\Delta \tilde{P}_{\alpha \beta}^{(3)}=16 \tilde{J} \sin \tilde{F}_{21} \sin \tilde{F}_{31} \sin \tilde{F}_{32}$ [12]. The overall matter contamination 
residing in $\Delta \tilde{P}_{\alpha \beta}$ is usually expected to be insignificant. The reason is simply that the terrestrial matter effects in $\tilde{P}\left(\nu_{\alpha} \rightarrow \nu_{\beta}\right)$ and $\tilde{P}\left(\nu_{\beta} \rightarrow \nu_{\alpha}\right)$, which both depend on the parameters $\left(a, a^{\prime}\right)$, may partly (even essentially) cancel each other in the T-violating asymmetry $\Delta \tilde{P}_{\alpha \beta}$. In contrast, $\tilde{P}\left(\nu_{\alpha} \rightarrow \nu_{\beta}\right)$ and $\tilde{P}\left(\bar{\nu}_{\alpha} \rightarrow \bar{\nu}_{\beta}\right)$ are associated respectively with $\left(+a,+a^{\prime}\right)$ and $\left(-a,-a^{\prime}\right)$, thus there should not have large cancellation of matter effects in the corresponding CP-violating asymmetries.

To summarize, let me remark that the strategy of this talk is to formulate the sum rules of neutrino masses and $\mathrm{CP}$ violation in a model-independent way. An obvious sum rule of neutrino masses is, of course,

$$
\tilde{m}_{0}^{2}+\tilde{m}_{1}^{2}+\tilde{m}_{2}^{2}+\tilde{m}_{3}^{2}=m_{0}^{2}+m_{1}^{2}+m_{2}^{2}+m_{3}^{2}+a+a^{\prime},
$$

which arises straightforwardly from Eqs. (2), (3) and (4). Following a lesson learnt from Heisenberg's matrix Quantum Mechanics, I have introduced the commutators of lepton mass matrices to describe the flavor mixing phenomenon of three active and one sterile neutrinos. It has been shown that the commutator defined in vacuum is invariant under terrestrial matter effects. An important consequence of this interesting result is the emergence of a set of model-independent sum rules for neutrino masses in two different media. I have also presented some useful sum rules for the rephasing-invariant measures of leptonic CP violation in the four-neutrino mixing scheme. A generic formula of T-violating asymmetries, which is applicable in particular to the future long-baseline neutrino oscillation experiments, has been derived and discussed.

\section{References}

[1] C. Jarlskog, Phys. Rev. Lett. 55 (1985) 1039; H. Fritzsch and Z.Z. Xing, Phys. Lett. B 353 (1995) 114; Nucl. Phys. B 556 (1999) 49; and references therein.

[2] Super-Kamiokande Collaboration, Y. Fukuda et al., Phys. Rev. Lett. 81 (1998) 1562; 81 (1998) 4279; http://www-sk.icrr.u-tokyo.ac.jp/dpc/sk/;

[3] LSND Collaboration, C. Athanassopoulos et al., Phys. Rev. Lett. 81 (1998) 1774.

[4] For a recent review with extensive references, see: H. Fritzsch and Z.Z. Xing, Prog. Part. Nucl. Phys. 45 (2000) 1; hep-ph/9912358.

[5] See, e.g., B. Autin et al., Report No. CERN-SPSC-98-30 (1998); M.G. Catanesi et al., Report No. CERN-SPSC-99-35 (1999); D. Ayres et al., physics/9911009; C. Albright et al., hep-ex/0008064; H. Chen et al., hep-ph/0104266; and references therein.

[6] Z.Z. Xing, Phys. Lett. B 487 (2000) 327; Phys. Rev. D 64 (2001) 073014.

[7] Z.Z. Xing, Phys. Rev. D 64 (2001) 033005.

[8] L. Wolfenstein, Phys. Rev. D 17 (1978) 2369; S.P. Mikheyev and A.Yu. Smirnov, Yad. Fiz. (Sov. J. Nucl. Phys.) 42 (1985) 1441.

[9] V. Barger, Y.B. Dai, K. Whisnant, and B.L. Young, Phys. Rev. D 59 (1999) 113010.

[10] P.F. Harrison and W.G. Scott, Phys. Lett. B 476 (2000) 349.

[11] A. Kalliomäki, J. Maalampi, and M. Tanimoto, Phys. Lett. B 469 (1999) 179.

[12] H. Fritzsch and Z.Z. Xing, Phys. Lett. B 517 (2001) 363; Phys. Rev. D 61 (2000) 073016; Phys. Lett. B 440 (1998) 313; Phys. Lett. B 372 (1996) 265. 